\title{
Rational Approximations for Irrational Functions using Collocating Continued Fraction Expansions
}

\author{
Philip D Olivier
}

February 14, 2021

\begin{abstract}
This paper is motivated by the need in certain engineering contexts to construct approximations for irrational functions in the complex variable $z$. The main mathematical tool that will be used is a special continued fraction expansion that cause the rational approximant to collocate the irrational function at specific important values of $z$. This paper introduces two theorems that facilitate the construction of the rational approximation.
\end{abstract}

\section{Introduction and Problem Statement}

The motivating problem is that of constructing rational approximations to an irrational function of $\mathrm{z}$ that are of interest in control engineering. The control engineering details are mostly unimportant as this paper focuses on the mathematical development of a new (nuanced?) continued fraction expansion that is useful in constructing such approximations.

A few words about the engineering context might be helpful. The real world evolves in continuous time, t. As computers and sensors have become smaller, more powerful, and less expensive, most objects that interact with the real word have become increasingly digital, i.e. they evolve in discrete time, k. Linear differential equations are very conveniently described using Laplace transforms, i.e. in the s-domain. Linear difference equations are most conveniently described using $\mathrm{z}$-transforms, i.e. in the z-domain. The mapping between the s-domain and the z-domain is

$$
z=e^{-s T}
$$

where $T$ is the sampling time. Inverting this mapping yields

$$
s=-\frac{\ln (z)}{T} .
$$

An example of an s-domain function being mapped into the z-domain might simultaneously help the reader by a) making things more concrete and b) pre- 
venting the reader from over simplifying. Consider mapping

$$
G(s)=\frac{s^{1.2}}{s^{2}+s^{0.75}+1}
$$

into the z-domain to get

$$
H(z)=[G(s)]_{s=-\ln (z) / T}=\frac{\left(-\frac{\ln (z)}{T}\right)^{1.2}}{\left(-\frac{\ln (z)}{T}\right)^{2}+\left(-\frac{\ln (z)}{T}\right)^{0.75}+1}
$$

Further details of the function $H(z)$ are unimportant. it is only important to note the combination of irrational features (i.e. fractional powers and $l n$ function) within a traditional fraction.

\subsection{Preview of Collocating CFEs}

The CFEs developed in this paper begin with levels similar to

$$
G(z)=a_{0}+\frac{1}{b_{1}+\frac{z}{b_{2}+\frac{z-1}{m_{3} z+b_{3}+(z-\hat{z})\left(z-\hat{z}^{*}\right) E_{3}}}}
$$

Notice that what occurs below the $z$ does not affect the value of the CFE at $z=0$; what happens below the $z-1$ does not affect on the value of the CFE at $z=1$ and what happens in $E_{3}$ does not affect the value of the CFE at $z=\hat{z}$ or $z=\hat{z}^{*}$. The values of the expressions $a_{0}, b_{1}, b_{2}, m_{3}$ and $b_{3}$ are chosen so that the CFE reproduces the values of $\mathrm{G}(\mathrm{z})$ at $z=0, z=1, z=\hat{z}$ and $z=\hat{z}^{*}$ as well as preserving the pole-zero excess if desired and possible possible.

It is natural to wonder why the collocating does not start until the second level. The first level provides the approximation the flexibility to produce (or avoid producing) improper functions (numerator order higher than that of denominator), bi-proper functions (numerator and denominator have the same order) and strictly proper functions (numerator order lower than denominator).

1. improper functions result when $a_{0}$ is chosen as a non-constant polynomial in $\mathrm{z}$.

2. bi-proper functions result when $a_{0} \neq 0$ is chosen as a constant

3. strictly-proper functions result when $a_{0}=0$ and $b_{1} \neq 0$

\section{CFE Theory}

Standard books on continued fraction expansions (CFEs) such as [?] contain material similar to the material found in this section. There are slight changes 
in notation as compared to that found in [?], some of the changes are due to the precise applications envisioned for the collocating CFEs. Collocating CFEs for sunction in the s-domain are discussed in [?].

There are two, equivalent, views of continued fractions. What they have in common is that in each case, an expression is continued via a linear fractional transformation. They both start as expressions in terms of a function $E_{i}$ and are continued via the substitution $E_{i}=1 /\left[\alpha_{i+1}+\beta_{i+1} E_{i+1}\right]$ where the $\alpha_{i+1}, \beta_{i+1}$ and $E_{i+1}$ can be real or complex numbers or perhaps more complex expressions, again, the reader is urged to not over simplify these in their mind. That is

$$
F(z)=f_{i}\left(z, E_{i}\right)=f_{i+1}\left(z, E_{i+1}\right)
$$

Further, they can be truncated by setting $E_{i}=0$ producing the $i^{\text {th }}$ approximant

$$
F(z) \approx f_{i}(z, 0)
$$

The first view, herein called the traditional view, motivates the term "continued fraction". The second view is more useful in constructing collocating CFEs..

\subsection{Traditional View of CFEs}

Everything in this subsection is slightly modified from what can be found in [?]. The term continued fraction expansion comes from the traditional description of an expression of the form

$$
F(z)=\alpha_{0}+\beta_{0} E_{0}=\alpha_{0}+\frac{\beta_{0}}{\alpha_{1}+\frac{\beta_{1}}{\alpha_{2}+\frac{\beta_{2}}{\alpha_{3}+\ldots}}}
$$

where the $\alpha$ 's and $\beta$ 's can be real or complex numbers or expressions. This CFE can be built, step-by-step, as follows

$$
F(z)=\alpha_{0}+\beta_{0} E_{0}=\alpha_{0}+\frac{\beta_{0}}{\alpha_{1}+\beta_{1} E_{1}}
$$

with $E_{1}=\frac{1}{\alpha_{2}+\beta_{2} E_{2}}$ and $E_{i}=\frac{1}{\alpha_{i+1}+\beta_{i+1} E_{i+1}}$. This leads to the expanding expressions

$$
F(z)=\alpha_{0}+\frac{\beta_{0}}{\alpha_{1}+\beta_{1} E_{1}}=\alpha_{0}+\frac{\beta_{0}}{\alpha_{1}+\frac{\beta_{1}}{\alpha_{2}+\beta_{2} E_{2}}}=\alpha_{0}+\frac{\beta_{0}}{\alpha_{1}+\frac{\beta_{1}}{\alpha_{2}+\frac{\beta_{2}}{\alpha_{3}+\beta_{3} E_{3}}}}=\ldots
$$

When starting with a rational expression, the simplest way to construct a continued fraction expansion is to invert the original rational expression and 
perform long division. The quotient is the $\alpha_{i}$ and the CFE is continued by treating the remainder divided by the original numerator in like manner. The CFE will eventually terminate on its own. These CFEs turn out to be computationally efficient, about as computationally efficient as the most efficient computational method for the original rational expression.

\subsection{Alternate View, A, B, C, D view}

The alternate view is called herein the $\mathrm{ABCD}$ view, (also see [?]). In this view, the CFE could begin with

$$
F(z)=\alpha_{0}+\beta_{0} E_{0}=\frac{A_{0}+B_{0} E_{0}}{C_{0}+D_{0} E_{0}}, \quad A_{0}=\alpha_{0}, B_{0}=\beta_{0}, C_{0}=1, D_{0}=0
$$

and for integer $i \geq 1$ substitute $E_{i}=\frac{1}{\alpha_{i+1}+\beta_{i+1} E_{i+1}}$ just as before. The difference is that at each substitution, the CFE is partially collapsed to produce

$$
F(z)=\frac{A_{i}+B_{i} E_{i}}{C_{i}+D_{i} E_{i}}
$$

Where the $A_{i}, B_{i}, C_{i}$ and $D_{i}$ can be conveniently, recursivly computed. The recursive computation is proven the the following theorem. An analogous recursive equation theorem can be found in [?].

Theorem 1 Recursive Computation Theorem: A fractional expression of the form

$$
F=\frac{A_{i}+B_{i} E_{i}}{C_{i}+D_{i} E_{i}}
$$

can be continued by substituting $E_{i}=\frac{1}{\alpha_{i+1}+\beta_{i+1} E_{i+1}}$ to produce the fractional expression

with

$$
F=\frac{A_{i+1}+B_{i+1} E_{i+1}}{C_{i+1}+D_{i+1} E_{i+1}}
$$

$$
\begin{gathered}
A_{i+1}=\alpha_{i+1} A_{i}+B_{i} \\
B_{i+1}=\beta_{i+1} A_{i} \\
C_{i+1}=\alpha_{i+1} C_{i}+D_{i} \\
D_{i+1}=\beta_{i+1} C_{i}
\end{gathered}
$$

Proof: Algebraic substitution, followed by algebra.

Notice that these recursive equations are independent of the starting values of $A_{0}, B_{0}, C_{0}$ and $D_{0}$.

One of the advantages of the A,B,C,D view is that at each step the CFE is partially and incrementally "collapsed" producing the polynomials in the partial rational approximation. Related to this advantage is that setting $E_{i+1}=0$ produced the $i^{\text {th }}$ rational approximant

$$
F(z) \approx F_{i}(z)=\frac{A_{i}(z)}{B_{i}(z)}
$$




\subsection{Two new Results.}

Up to this point, the discussion has focused on reviewing well known results. In this subsection two results are presented that are tools in constructing collocating CFEs. As such they are not as directly related to classical CFE theory. Both provide expressions for $\alpha_{i+1}+\beta_{i+1} E_{i+1}$

The derivation of the first expression is produced by rearranging

$$
\begin{gathered}
F(z)=\alpha_{0}+\frac{\beta_{0}}{\alpha_{1}+\beta_{1} E_{1}} \\
\alpha_{1}+\beta_{1} E_{1}=\frac{\beta_{0}}{F(z)-\alpha_{0}}=\hat{G}_{1}
\end{gathered}
$$

which makes it easy to determine $\alpha_{1}$ and $\beta_{1}$. So now assume we have determined $\alpha_{1}$, and $\beta_{1}$. How to choose these will be discussed later, first a recursive relation is derived to aid in choosing any $\alpha_{i}$ and $\beta_{i}$.

The following Lemma shows an alternate expression for $\alpha_{k+1}+\beta_{k+1} E_{k+1}$ in terms of the ABCD representation.

Lemma 1: Same assumptions as in Theorem 1. Suppose that

$$
E_{i+1}=\frac{1}{\alpha_{i+1}+\beta_{i+1} E_{i+2}}
$$

then

$$
\alpha_{i+1}+\beta_{i+1} E_{i+1}=\frac{B_{i}-F D_{i}}{F C_{i}-A_{i}}
$$

Now assume this is true for some $k$ (it is for $k=1$ ) is it true for $k+1$ ? Assume $\alpha_{k}$ and $\beta_{k}$ are known

$$
\hat{G}_{k}=\alpha_{k}+\beta_{k} E_{k}=\alpha_{k}+\frac{\beta_{k}}{\alpha_{k+1}+\beta_{k+1} E_{k+1}}
$$

Solve for $\alpha_{k+1}+\beta_{k+1} E_{k+1}$ in terms of $\hat{G}_{k}$ and define $\hat{G}_{k+1}$

$$
\alpha_{k+1}+\beta_{k+1} E_{k+1}=\frac{\beta_{k}}{\hat{G}_{k}-\alpha_{k}}=\hat{G}_{k+1}
$$

\section{Function Approximation with ABCD CFEs}

In addition to the two results in equations (1) and (2) in the last subsection above, the new contributions of his paper are contained in this section. Even these are not totally new, since precursor results can be found in [?] as applied to Laplace domain functions. Consider approximating $F(z)$

$$
F(z)=\frac{A_{0}+B_{0} E_{0}}{C_{0}+D_{0} E_{0}}
$$


What properties of $F(z)$ should be retained in its approximation? The focus of this work is to retain the values at certain specified values of $z$, i.e. to cause the approximation to collocate with the original. Other properties might be important to retain. For the purposes of this discussion the most important features to be retained are:

1. approximate pole-zero excess

2. $F(r), r=0$, or $r=1$, or $r$ any real number

3. $F\left(e^{ \pm j \omega}\right), \omega$ a frequency

4. etc

The first three are of primary importance in this work. The z-transforms of many functions are rational. In addition, many have the property $F(0)=0$, meaning that their numerators have an uncancelled factor of $z$. In control engineering applications, the value of $F(1)$ is also important because it determines important steady-state behavior. The value of $F\left(e^{ \pm j \omega}\right)$, i.e. at a pair of points on the unit circle, determines the behavior of the system for a sinusoidal input, and hence is of importance.

The near term objective is to construct, step-by-step, an approximation to $F(z)$ that preserves $F(0), F(1)$, and $F\left(e^{ \pm j \omega}\right)$.

$$
F(z)=\alpha_{0}+\beta_{0} E_{0}, \quad A_{0}=\alpha_{0}, B_{0}=\beta_{0}, C_{0}=1, D_{0}=0
$$

to achieve a bi-proper approximation choose $\alpha_{0} \neq 0, \alpha_{0} \neq F(0), \alpha_{0} \neq F(1)$, $\beta_{0}=1$.

To collocate at $z=0$, extend the CFE, choosing $\beta_{1}=z$, by substituting $E_{0}=1 /\left[\alpha_{1}+z E_{1}\right]$ to get

$$
F(z)=\alpha_{0}+\frac{\beta_{0}}{\alpha_{1}+z E_{1}}=\frac{A_{1}+B_{1} E_{1}}{C_{1}+D_{1} E_{1}}
$$

Evaluate at $\mathrm{z}=0$ to get

$$
\alpha_{1}=\frac{\beta_{0}}{F(0)-\alpha_{0}}
$$

Use the recursive equations to update the $\mathrm{ABCD}$ representation

$$
A_{1}=A_{0} \alpha_{1}+B_{0} \quad B_{1}=z A_{0} \quad C_{1}=\alpha_{1} C_{0}+D_{0} \quad D_{1}=z C_{0}
$$

To collocate at $z=1$, extend the CFE, choosing $\beta_{2}=z-1$, substituting $E_{1}=1 /\left[\alpha_{2}+(z-1) E_{2}\right]$, and using Lemma 1 , to get

$$
\alpha_{2}+(z-1) E_{2}=\frac{B_{1}-F D_{1}}{F C_{1}-A_{1}}
$$

evaluate this at $z=1$ to get

$$
\alpha_{2}=\left[\frac{B_{1}-F D_{1}}{F C_{1}-A_{1}}\right]_{z=1}
$$


Now use the recursive equations to update the ABCD representation.

To collocate at $z=e^{ \pm j \omega}$, extend the CFE, choosing $\beta_{3}=\left(z-e^{j \omega}\right)(z-$ $\left.e^{-j \omega}\right)=z^{2}-2 \cos (\omega)+1$, choose $\alpha_{3}=m_{3} z+b_{3}$ for real valued $m_{3}$ and $b_{3}$, use Lemma 1 to get

$$
m_{3} z+b_{3}+\left(z^{2}-2 \cos (\omega)+1\right) E_{3}=\frac{B_{2}-F D_{2}}{F C_{2}-A_{2}}
$$

Evaluate at $z=e^{j \omega}$

$$
m_{3}[\cos (\omega)+j \sin (\omega)]+b_{3}=\left[\frac{B_{2}-F D_{2}}{F C_{2}-A_{2}}\right]_{z=e^{j \omega}}
$$

Solve for $m_{3}$ by taking imaginary parts of both sides of the equation, and for $b_{3}$ by taking real parts of both sides of the equation to get

$$
\begin{gathered}
m_{3}=\frac{1}{\sin (\omega)} \operatorname{Im}\left(\left[\frac{B_{2}-F D_{2}}{F C_{2}-A_{2}}\right]_{z=e^{j \omega}}\right) \\
b_{3}=\operatorname{Re}\left(\left[\frac{B_{2}-F D_{2}}{F C_{2}-A_{2}}\right]_{z=e^{j \omega}}\right)-m_{3} \cos (\omega)
\end{gathered}
$$

Theorems 2 and 3 formalize the process of collocating at real values of $z$ and at $z$ 's on the unit circle. The proofs are omitted because that would be repeat the work with by simply changing the numeric subscripts to symbolic subscripts..

Theorem $2 z=r$ Collocation Theorem If $F(z)=\frac{A_{i}+B_{i} E_{i}}{C_{i}+D_{i} E_{i}}, A_{i}, B_{i}, C_{i}$, and $D_{i}$ are known, and if $E_{i}=\frac{1}{\alpha_{i}+\beta_{i} E_{i+1}}$ then to collocate at $z=r$ choose $\beta_{i+1}=(z-r)$ and $\alpha_{i+1}=\frac{B_{i}-D_{i} F(r)}{C_{i} F(r)-A_{i}}$

Theorem $3 \hat{z}=e^{j \hat{\omega}}$ Collocation Theorem. Same assumptions as Theorem 2. The continued fraction can be extended so that it collocates at $\hat{z}=e^{j \hat{\omega}}$ by choosing

$$
E_{i}=\frac{1}{p_{i}(z)+\left(z^{2}-2 \cos (\hat{\omega}) z+1\right) E_{i+1}}
$$

with

$$
\begin{gathered}
p_{i}(z)=m_{i} z+b_{i} \\
m_{i}=\frac{1}{\sin (\hat{\omega})} \operatorname{Im}\left(\frac{B_{i}(z)-D_{i}(z) F(z)}{C_{i}(z) F(z)-A_{i}(z)}\right)_{z=e^{j \hat{\omega}}} \\
b_{i}=\operatorname{Re}\left(\frac{B_{i}(z)-D_{i}(z) F(z)}{C_{i}(z) F(z)-A_{i}(z)}\right)_{z=e^{j \omega}}-\cos (\hat{\omega}) m_{i+1}
\end{gathered}
$$




\section{Discussion and Conclusions}

An approach to $\mathrm{CFE}$ construction that causes the CFE to collocate with the original function is presented, the discussion included several theorems/results that aid in the construction.

Lemma 1 is one of the key results. It is, given $F, A_{i}, G_{i}, \mathrm{C}_{i}$, and $D_{i}$ for $i=0,1, \ldots k$

$$
\alpha_{k+1}+\beta_{k+1} E_{k+1}=\frac{B_{k}-F D_{k}}{F C_{k}-A_{k}}
$$

Using this result, it is possible to do many things not discussed in this paper. For instance, collocation at many points could be achieved by choosing $\alpha_{k+1}$ to be the Lagrange collocating polynomial (see [?]) for a set of points and the corresponding $\beta_{k+1}$ to be the corresponding polynomial that evaluates to zero at each of the points in the set.

Another observed application is that when ready to truncate the the CFE, one could arbitrarily set $\beta_{k+1} E_{k+1}=0$, and let $\alpha_{k+1}$ be an arbitrary polynomial that is chosed to minimize some cost functional like the mean square error cost functional or the min-max cost;

Yet another possibility is choose $\alpha_{k+1}$ to be the ratio of Laguerre Basis functions a la [?] and choose the coefficients to minimize the least square error.

While the key result mentioned at the top of this section is presented as a Lemma, in retrospect, it might be considered as the primary result.

A key issue of mathematical importance has been ignored, that of convergence. The goal of the CFEs discussed has been to produce a rational approximation that is finite order, so the CFEs are truncated at some point. Convergence results (see [?]) might be used to guide the choice of $\alpha$ 's and $\beta$ 's as the CFE is extended beyond the collocating levels. This is an area of future work.

\section{References}

[1] Wall, H. S. Analytic Theory of Continued Fraction Expansions, Dover reprint of the Van Nostrand, 1948 edition.

[2] Kalu, A. And Olivier, P.D. New CFE for System Approximation, Electronics Letters 1989.

[3] Hildebrand, F. B. Introduction to Numerical Analysis, Dover reprint of the McGraw-Hill Book Co. New York 1956 edition

[4] Olivier, P.D., (2021): System Identification Using Laguerre Basis Functions. TechRxiv. Preprint. https://doi.org/10.36227/techrxiv.13656701.v1

\section{About the author:}

Philip D. Olivier was a 1976 Richard V. Andree Award Winner ( $2^{\text {nd }}$ place) for his paper titled "Two Applications of Pseudo Inverses" which he wrote while a 
senior at Loyola University in New Orleans, LA. He earned his $\mathrm{PhD}$ in electrical engineering and MS in mathematics from Texas Tech University and his BS in Physics from Loyola University, New Orleans. Most of his professional career was spent teaching electrical engineering including stints as department chair and Assistant Dean as well as serving in other administrative posts. His research interests have always tilted toward the mathematical side of engineering, most particularly control theory and more generally in mathematical systems theory. He has done funded research for various private and governmental entities such as NASA, the US Air Force, Hyundai, and Ford Motor Co.

1533 E Federal Heights Dr.

Salt Lake City, UT 84103

philip_d_olivier@ieee.org 